\title{
Quantum Confinement Effects on Physical Properties of ZnTe Spherical Quantum Dots
}

\author{
L. LAKhAL ${ }^{a, b}$, F. MEZRAG ${ }^{a, b, *}$ AND N. BOUARISSA ${ }^{a, b}$ \\ ${ }^{a}$ Laboratory of Materials Physics and Its Applications, University of M'sila, 28000 M'sila, Algeria \\ ${ }^{b}$ Physics Department, Faculty of Science, University of M'sila, 28000 M'sila, Algeria
}

Using a pseudopotential approach, the present study focuses on the effects of quantum confinement on physical properties of zinc-blende ZnTe spherical quantum dots. The size dependence of all features being considered here has been analyzed and discussed. Our results show that the effects of quantum confinement change the physical properties of nanostructured ZnTe dramatically relative to the bulk values. This provided with the possibility for obtaining new physical properties that was not provided by the bulk material.

DOI: 10.12693/APhysPolA.137.451

PACS/topics: quantum dots, nanostructured ZnTe, quantum confinement

\section{Introduction}

In the last decades, chalcogenide semiconducting materials have attracted much attention [1-5]. This is essentially due to their enhanced material hardness. Among these materials, ZnTe is a semiconductor with a direct band-gap of $2.23-2.25 \mathrm{eV}$ [6]. Typically, it has a cubic crystal structure. This material of interest found several applications which include solid state laser devices, photovoltaic devices, thin films, detectors, and so on. This material can also be used as a component of ternary alloys [7-10].

The new class of materials formed by semiconductor nanostructures has found a wide ensemble of possible applications. As a matter of fact, the electronic and optical properties of nanostructured materials such as semiconductor quantum dots are intermediate between those of discrete molecules, and those of bulk semiconductors. This makes them very important in the technological applications such as light emitting diodes, quantum computing, diode lasers, solar cells, and medical imaging [11-13].

In this work, the electronic energy levels and optical properties of ZnTe spherical quantum dots are studied. Features such as energy band-gap and refractive index have been reported and their dependence on the quantum dot size has been examined and discussed.

\section{Computational method}

The computations are carried out using the empirical pseudo-potential method (EPM) [14-16]. The approach even at the nanoscale has proven to yield good results [17-20]. The pseudopotential form factors (PPFFs) are determined by fitting the atomic form factors to

*corresponding author; e-mail: fadila.mezrag@univ-msila.dz experiment. The optimization of the PPFFs is done using a non-linear least-squares method as described in Refs. [21-23].

For studying quantum dots, we have reduced the dimensionality of the environment of electrons to a zero dimension. A proper boundary conditions have been imposed on the wave functions, which depend on both the size and shape of the quantum dots. The quantum confinement effect on the fundamental band-gap of $\mathrm{ZnTe}$ quantum dots is determined using the same approach as that used in Refs. [24, 25]. The electron and heavy hole effective masses of bulk ZnTe are obtained using a methodology similar to that used by Bouarissa [26].

\section{Results and discussion}

Figure 1 displays the variation of the direct $\Gamma-\Gamma$ energy band-gap as a function of the size for zinc-blende ZnTe quantum dots with a spherical shape. The radius of the quantum dot is taken to be in the range $1-5 \mathrm{~nm}$. Note that as the quantum dot radius is reduced, the $\Gamma-\Gamma$ band-gap increases. The increase becomes more important as the size is further reduced. The same qualitative behavior has been reported for other nanostructured binary semiconductors spherical quantum dots [20, 27]. The trend in $\Gamma-\Gamma$ energy band-gap versus the quantum dot radius is attributed to the quantum confinement effect which becomes more important as the size of the quantum dots is reduced. When the quantum dot size is much higher than the exciton Bohr radius (which is $6.7 \mathrm{~nm}$ for $\mathrm{ZnTe}$ compound), the effect of the dielectric confinement becomes weak. In that case the $\Gamma-\Gamma$ band-gap tends towards retrieving the value of $2.27 \mathrm{eV}$, which is the value of the bulk. This is in accord with our findings.

The refractive index $n$ of semiconductors is a fundamental parameter which characterizes their optical properties [28-31]. In the current contribution $n$ has been calculated for ZnTe spherical quantum dots using different models, namely the Hervé and Vandamme model [32], 


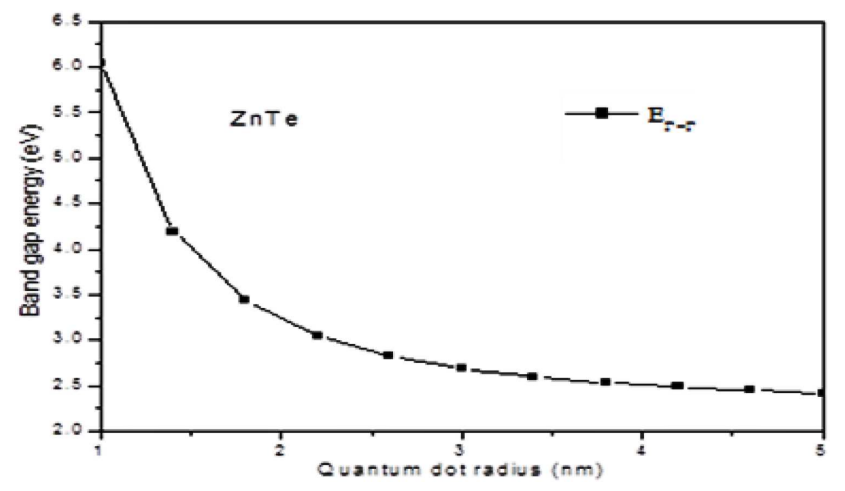

Fig. 1. Direct band gap $\Gamma-\Gamma$ in nanosized ZnTe versus quantum dot radius.

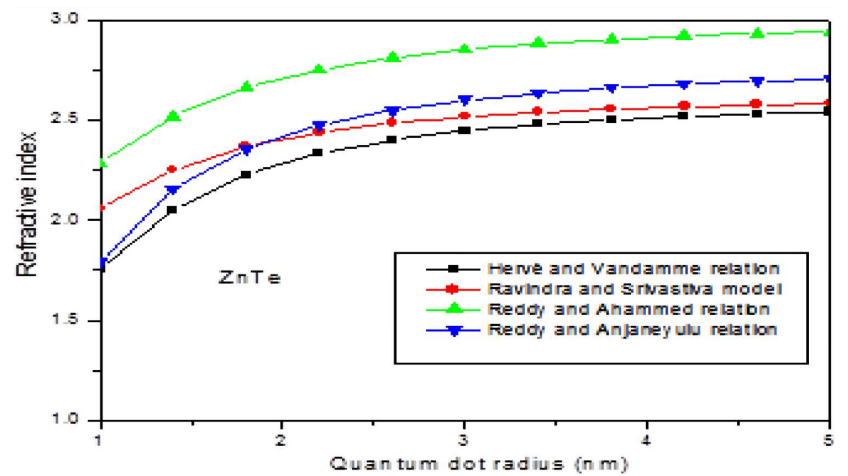

Fig. 2. Refractive index in nanosized $\mathrm{ZnTe}$ versus quantum dot radius using different models.

the Moss model [33] as revised by Ravindra and Srivastava [34], the Reddy and Ahammed model [35], and the Reddy and Anjaneyulu model [36]. Our results are illustrated in Fig. 2. We observe that by reducing the size of the quantum dot, $n$ for ZnTe quantum dots decreases. The ratio of the decrease becomes more important when approaching $1 \mathrm{~nm}$. Qualitatively, the same behaviour has been shown for all used models of interest. From the quantitative point of view, and due to the lack of data in the literature to compare with in the nanoscale $n$ has been compared with that of 2.72 reported in Ref. [37] for bulk ZnTe. Based on our results obtained from the different used models for bulk ZnTe, the best agreement is reached when the model of Gupta and Ravindra is used where $n$ is found to be 2.68 .

\section{Conclusion}

We have studied the size dependence of the direct $\Gamma-\Gamma$ energy band-gap and refractive index of zinc-blende ZnTe quantum dots with a spherical shape. The quantum dot radius was considered to be in the range $1-5 \mathrm{~nm}$. The computations were carried out using essentially a pseudopotential approach. The features of interest were found to be highly modified relative to the bulk values. This is attributed to the confinement effect.

\section{References}

[1] A. Tomasulo, M.V. Ramakrishna, J. Chem. Phys. 105, 3612 (1996).

[2] R.J. Nelmes, M.I. McMahon, Semicond. Semimetal. 54, 145 (1998).

[3] F. Benmakhlouf, N. Bouarissa, Int. J. Mod. Phys. B 20, 4807 (2006).

[4] L. Hannachi, N. Bouarissa, Physica B 404, 3650 (2009).

[5] S. Adachi, K. Sato, Jpn. J. Appl. Phys. 31, 3907 (1992).

[6] S. Ferahtia, S. Saib, N. Bouarissa, Int. J. Mod. Phys. $B$ 30, 1650147 (2016)

[7] S. Adachi, T. Kimura, Jpn. J. Appl. Phys. 32, 3496 (1993).

[8] S. Stolyarova, F. Edelman, A. Chack, et al., J. Phys. D Appl. Phys. 41, 065402 (2008).

[9] N. Bouarissa, Physica B 399, 126 (2007).

[10] A. Gueddim, S. Zerroug, N. Bouarissa, J. Lumin. 135, 243 (2013).

[11] R.C. Ashoori, Nature 379, 413 (1996).

[12] C.B. Murray, C.R. Kagan, M.G. Bawendi, Ann. Rev. Mater. Sci. 30, 545 (2000).

[13] H.Y. Ramirez, J. Flórez, A.S. Camacho, Phys. Chem. Chem. Phys. 17, 23938 (2015).

[14] M.L. Cohen, J.R. Chelikowsky, Electronic Structure and Optical Properties of Semiconductors, SpringerVerlag, Berlin 1989.

[15] R.M. Martin, Electronic Structure: Basic Theory and Practical Methods, Cambridge University Press, 2004.

[16] N. Bouarissa, Mater. Sci. Eng. B 86, 53 (2001).

[17] G. Bester, J. Phys. Condens. Matter 21, 023202 (2009).

[18] P.R.C. Kent, L. Bellaiche, A. Zunger, Semicond. Sci. Technol. 17, 851 (2002).

[19] N. Bouarissa, J. Comput. Theor. Nanosci. 10, 1284 (2013).

[20] F. Mezrag, N. Bouarissa, M. Boucenna, Optik 127, 1167 (2016)

[21] T. Kobayasi, H. Nara, Bull. Coll. Med. Sci. Tohoku Univ. 2, 7 (1993)

[22] K. Kassali, N. Bouarissa, Mater. Chem. Phys. 76 , 255 (2002).

[23] N. Bouarissa, M. Boucenna, Phys. Scr. 79, 015701 (2009).

[24] A. Gueddim, T. Eloud, N. Messikine, N. Bouarissa, Superlatt. Microstruct. 77, 124 (2015).

[25] R. Ragan, Ph.D. Thesis, California Institute of Technology, Pasadena 2002.

[26] N. Bouarissa, J. Phys. Chem. Solids 67, 1440 (2006).

[27] N.H.M. Al Wadiy, N. Bouarissa, M.A. Khan, Phys. Scr. 84, 015704 (2011).

[28] S. Adachi, J. Appl. Phys. 61, 4869 (1987). 
[29] N. Bouarissa, Mater. Chem. Phys. 72, 387 (2001).

[30] N.M. Ravindra, P. Ganapathy, J. Choi, Infrared Phys. Technol. 50, 21 (2007).

[31] F. Mezrag, W. Kara Mohamed, N. Bouarissa, Physica B 405, 2272 (2010).

[32] P. Hervé, L.K.J. Vandamme, Infrared Phys. Technol. 35, 609 (1994).

[33] T.S. Moss, Proc. Phys. Soc. B 63, 167 (1950).
[34] N.M. Ravindra, V.K. Srivastava, Infrared Phys. 19 603 (1979).

[35] R.R. Reddy, Y.N. Ahammed, Infrared Phys. Technol. 36, 825 (1995).

[36] R.R. Reddy, S. Anjaneyulu, Phys. Status Solidi B 174, K91 (1992).

[37] D.W. Palmer, www.semiconductors.co.uk, 2008. 